\title{
MEMES CLASSIFICATION SYSTEM USING COMPUTER VISION AND NLP TECHNIQUES
}

\author{
Rachana Jadhav \\ Department of Computer Science and Engineering \\ Walchand College of Engineering (An Autonomous Institute) \\ Sangli, India \\ Prof. Vikas. N. Honmane \\ Department of Computer Science and Engineering \\ Walchand College of Engineering (An Autonomous Institute) \\ Sangli, India
}

\begin{abstract}
A meme is a culturally relevant, and brief form of media that raise a content over the internet. Now a days posting a meme is popular communication medium, due to its multimodal nature. Postings of hateful memes or fooling, cyberbullying are growing gradually. Meme takes a major part in forming people's trust and perspective. Meme can be quickly post by anybody, and its integrity stands compromised. Hateful and aggressive matter detection have been largely traversed in a form such as text or image. And Memes complicate the task, because some meme can have a good caption and normal pictures, but if combined in some way, they can become offensive. So, it is required to fuse both modality to identify whether a given meme is hateful or not. So here for text classification we found the sequential model like Bi-LSTM and for image we will go with CNN. Late fusion technique is used to combine the image and text mode with EX-OR method to investigate its effectiveness.
\end{abstract}

Index Terms-Hateful meme, Deep Learning, Natural Language Processing, Late fusion, Image Processing, Bi-LSTM, EX-OR.

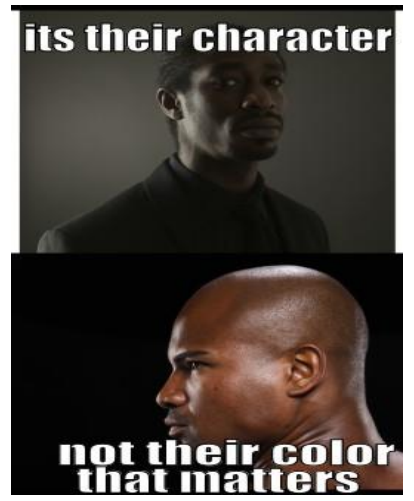

Fig. 1. Sample meme

\section{INTRODUCTION}

Now a days social media be it Facebook, Instagram, Twitter or any other social media has gained a lot of enticement since its beginning. Day by day the content generation is growing rapidly. Almost half population of society is present on social media. Most of the present day memes have more impact on people as they are structurally apart from longer form of media. Memes become successful because they are Familiar, short, brief, culturally relevant and quickly understood by the target audience.

Memes can be in many forms like

- Image Dominant: where textual data is less.

- Text Dominant: where textual data is huge

- Text and Image Dominant: where textual and image data are same.

Recently the most central usage of memes to be of images combined with text. Irrespective of the type of meme, the meme may get modified, rehashed or recreated while inter- acting through social media network. In this age memes are decorated fancy pictures that are put forward to be diverting, often as a way to freely disrespect individual control. Additional memes can be spoken recordings, and few memes have a more logical subject. So due to the massive use of social networks, negative impacts need to be automatically limited.

This project serves to propose a technique which uses text mode and image mode processing to classify the memes as hateful or non-hateful and stop spreading hate across internet. This work aims to conduct thorough approaches in computer vision, deep learning and natural language processing.

\section{LITERATURE REVIEW}

As there was need to detect offensive content author 
has created the MultiOFF dataset .Later developed a classifier for this task. An early fusion technique was used to merge the text and image mode and compare it with baseline models of image and text to look over its efficiency [1].They present a strong non paranormal perspective which is capable to learn and transform text and vision features into the cumulative density function space. Along with, they show that their model able to perform better with various effective baselines. Moreover, they also showed a progress in generating memes from raw images [2].

The author created an attention model based on semantic similarity to overcome the limitations of BERT .Developed a BERT-based model that does a better job than most models in the MovieQA problem. In order to solve MCQ, the author first extracts sentences from large text, which makes it easier to answer MCQ questions [3].To differentiate between profanity, hate speech and other texts author has applied Some lexical features and applied a Support Vector Machine classifier to set up a measure. A character 4-gram model works well for this task [4].

Extraction and preprocessing of text, image and face encoding is done. Author performed four different groups of classifiers but LSTM model perform well than other text models and for vision DNN models show better advancement [5].Author propose a method to analyze memes based on sentiments of meme. Memes with emotions like happy, angery, or other kind of emotions are collected. Visual features are extracted MATLAB functions and textual features using OCR.J48 algorithm worked accurately on the memes [6].

This paper shows a various ways like concatenation bilinear transformation and gated summation to fuse text and photo signal with further improvement [7].Two architectures are evaluated for detection of objects in text for visual question answering. A late fusion architecture where text and image are encoded separately before fuse result. , And early fusion B2T2 model where visual features are placed on the same level as input word tokens. B2T2 is highly effective [8].

This paper presents a new challenging dataset for identification of hateful speech in multimodal memes. It is established by adding some meme example in such a way that unimodal classifier would fight to classify them. Also some baseline are provided for both models. But existing methods fail to reach at performance. So this pointing the challenge to community [9].

\section{METHODOLOGY}

Unimodal Classification are easy, but when we are working on Multimodal classification it becomes tricky.
Because we have to combine data from different modalities.

Here we are going with Late Fusion approach late fusion will classify content for both text and image mode before trying to fuse the results.

\section{A. Memes pre-processing}

\section{Textual Data Processing}

The first step before feeding text data to any machine learning algorithm involves text pre-processing in order to clean the data.

- Remove the unnecessary tags.

- Remove any punctuation or a limited set of special characters like, or, etc.

- Check if the word is not alpha-numeric and should made up of English letters.

- Convert into lowercase form.

- Remove Stop-words which do not add any significance, like is, am, the etc.

- Convert into short into full as these words are not registered in dictionaries. As a result, the main problem is that the same word may have different meanings, depending on the situation.

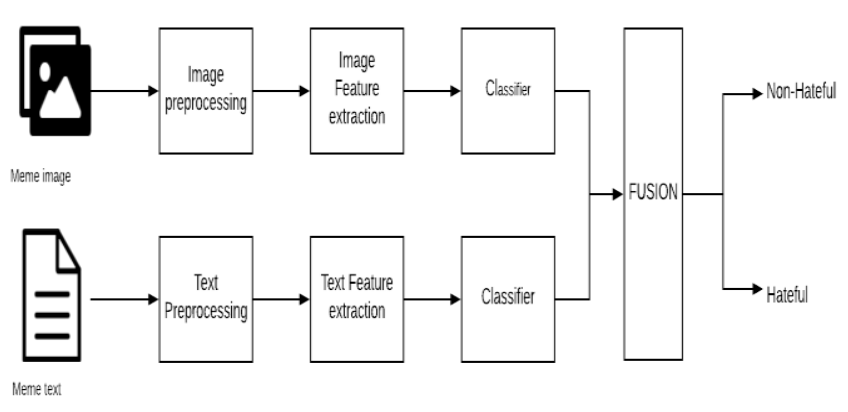

Fig. 2. Late Fusion

\section{Image Data Processing}

- Resizing: The memes collected are of different sizes so need to resized uniformly before being used as input to model therefore, we should establish a base size for all images fed into our algorithms.

- Noise Reduction: Various filters can be used. Most commonly used filters are Mean filter, Gaussian Filter, Median Filter will use one which will improve the system functioning.

- RGB2Gray: Converting color image to gray scale image. 


\section{B. Memes Feature Extraction}

\section{Textual Feature Extraction:}

One hot encoding work straight with categorical variables with representing them as binary vectors. Each word is written or encoded as a one-hot vector, and each one-hot vector is unique, which allows a word to be uniquely identified by its one-hot vector. The reverse is also true, that is two words do not have the same one-hot vector representation. In this respect we can assign 'non- hateful' an integer value of 0 and 'hateful' the integer value of 1 . And its corresponding binary vector $[1,0]$ for 'nonhateful' and $[0,1]$ for 'hateful. Therefore, we represent each word and character in the text data as a unique vector, which includes numeric data ( 1 and 0 ) as its components. A word is represented as a vector so that a list of words can be represented in a sentence as a vector array or matrix. If we have a list of sentences in which words are hot-encoded, the result is a matrix whose elements are matrices. Then we get a three-dimensional tensor that can be passed to the neural network.

\section{Memes Classification}

\section{1) Image Classification using $\mathrm{CNN}$ :}

CNN image classification takes input images, processes them and classifies them into specific categories (such as dogs, cats, tigers, lions). The computer treats the input image as an array of pixels and depends on the image resolution. You will see $\mathrm{H} \times \mathrm{W} \times \mathrm{D}(\mathrm{H}=$ height, $\mathrm{W}=$ width, $\mathrm{D}=$ size). For example, a $6 \times 6 \times 3$ matrix image of RGB (3 refers to RGB values) and a $4 \times 4 \times 1$ matrix image of grayscale Technically speaking, for the deep learning CNN model used for training and testing, each input image goes through a series of convolutional layers with filters (kernels), pools, and fully connected layers (FC), and the softmax function is applied For objects with probability values to be classified are between 0 and 1 . The below figure is a complete flow of CNN to process an input image and classifies the objects based on values.

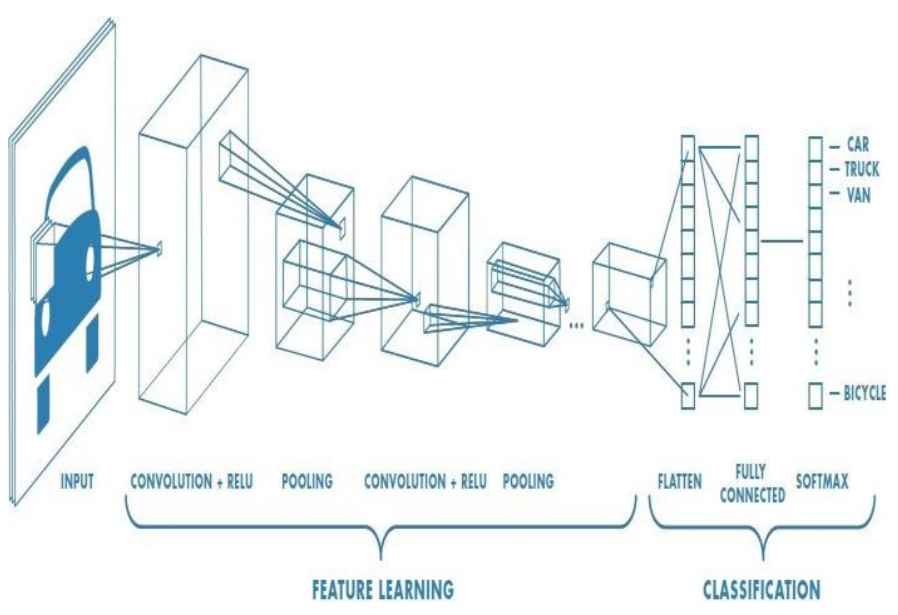

Fig. 3. CNN

2) Text classification using Bi-LSTM

Bidirectional LSTM or bi-LSTM is a sequence processing model that consists of two LSTMs: one receives input data in the forward direction, and the other receives input data in the opposite direction. Bi-LSTM effectively increases the amount of information available to the network by improving the context available to the algorithm. (For example, know which word immediately precedes a word in a sentence) The Bi-LSTM neural network consists of LSTM modules that work bi-directionally, including past and future contextual in- formation. Bi-LSTM can learn long-term dependence without obtaining repeated contextual information, so it exhibits excellent performance in sequential modeling tasks and is widely used in text classification. In the LSTM network, the Bi-LSTM network has two parallel layers, which propagate in two directions by passing forward and backward to capture the dependencies in the two contexts. The structure of the Bi-LSTM network is shown in Figure.

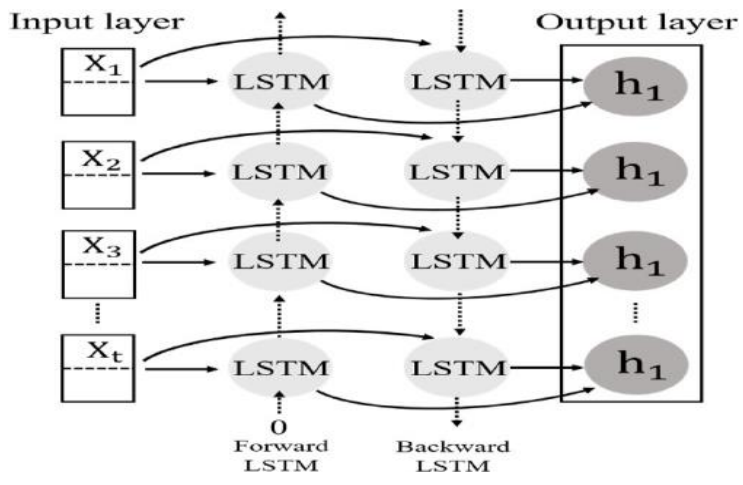

Fig. 4. Bi-LSTM 


\section{Fusion of Text and image}

Ex-OR Prediction: If any one of them be a text or image is hateful then it will result hateful. The following snippet of code will show you the exact idea that has been implemented:

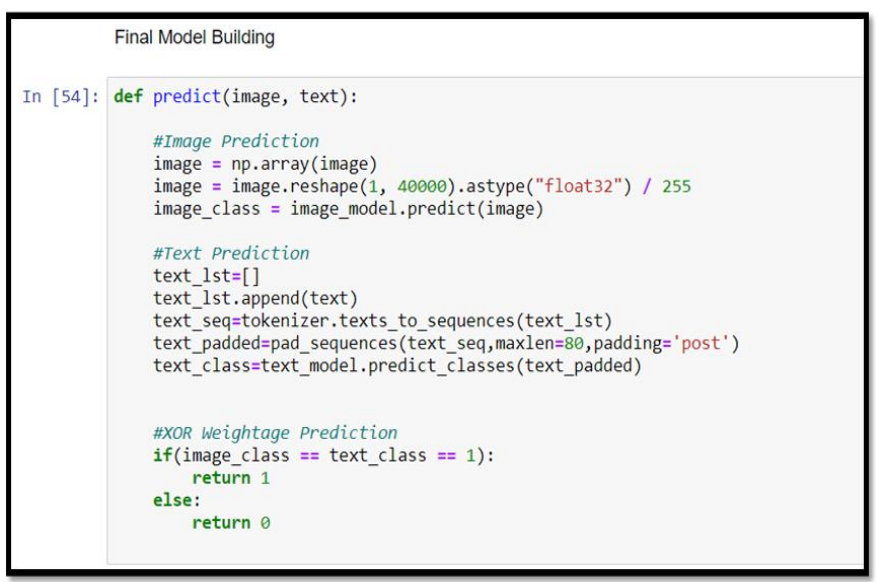

Fig. 5. Fusion

\section{SignificACE}

To stop spreading offensive content on online social media Also few brands are marketing with memes to connect with their followers or consumer on social media so it can be supportive in advertising and promotion or publicity of products and services. Some people said or done something mean or cruel to another person online so this system will be helpful for Cyber bullying monitoring. Trend analysis i.e. you can get information from past and you will come up with an idea for future in areas like, intellectual analysis, finding the popularity of a political party,

\section{RESULTS AND DISCUSSION}

We worked on hateful memes classification through approaches in Natural Language Processing and Deep learning with help of different libraries like NLTK, Keras, Tensorflow, SkLearn, Matplotlib, and Scikitplot to get work done. we have used a sequential model for text classification like Bi-LSTM which gives average accuracy of $87 \%$ And for

image Convolutional Neural Network is used with average accuracy of 35\%.Finally Late fusion of meme with EX-OR prediction of both the form that is text and image is done which gives $87 \%$ overall accuracy.

The most popular performance measures such as accuracy, precision, sensitivity, F1 Score are used to evaluate the performance of the proposed model.

- Accuracy $=$ Total No. of Correct Predicted $/$ Total No. of Observations

- Precision $=$ Total No. of correct predicted Category Observations / Total No. of Predicted Category Observations

- Recall $=$ Total No. of correct predicted Category Observations / Total No. of Actual Correct Category Observations

- F-Measure is the arithmetic mean of precision and recall.

Fig. 6. Confusion Matrix

The following GUI will give you the exact idea that has been implemented:

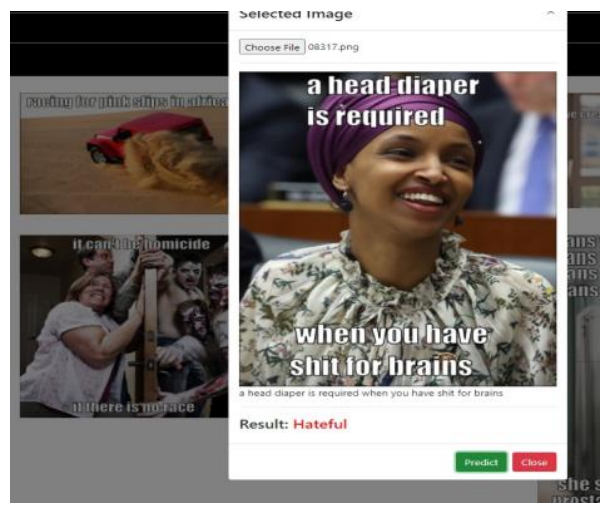

Fig. 7. Hateful

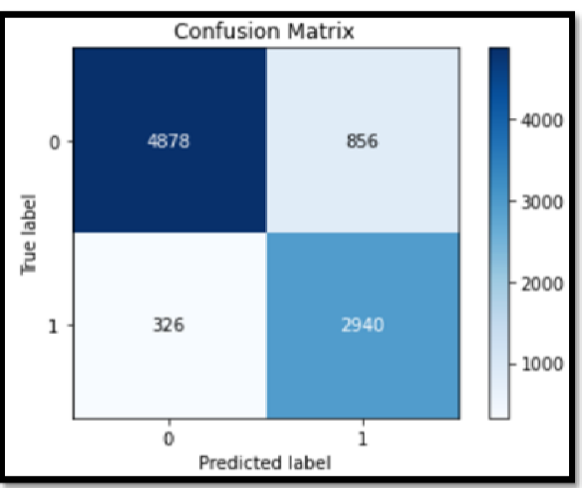




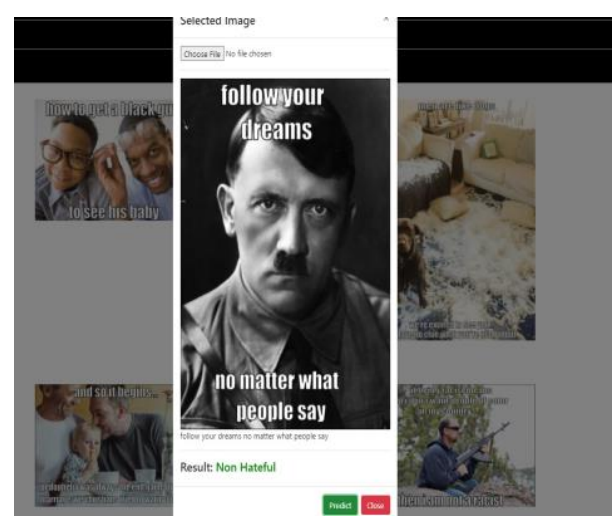

Fig. 8. Non-hateful

\section{CONCLUSION AND FUTURE WORK}

We proposed to investigate the important problem of memes classification system using computer vision and NLP techniques. Suggests a method that could be beneficial to classify memes with fix visual and textual features. Late Fusion will classify content for both text and image before trying to fuse the results. Eventually, further research and work to identify hateful meme is in progress and will give a more refined classification scheme for meme.

In the future, we plan to extend this work to other multimodal feature extraction methods to improve training on specific data sets. In addition, social media trends and patterns are changing rapidly, so it is necessary to capture memes in real time with respect to a particular domain so as to find the influential entities. This work can be extended to collect this data in real time and train deep learning models to identify hateful memes.

\section{REFERENCES}

[1] Bharathi Raja Chakravarthi,Shardul Suryawanshi Mihael Arcan, Paul Buitelaar, "Multimodal Meme Dataset (MultiOFF) for Identifying Of- fensive Content in Image and Text,"Language Resources and Evaluation Conference (LREC 2020), Marseille, 11-16 May 2020.

[2] William Yang Wang, Miaomiao Wen," I Can Has Cheezburger? A nonparanormal approach to combining textual and visual information for predicting and generating popular meme descriptions" In: The 2015 Annual Conference of the North American Chapter of the ACL, pp. 355365 (2015).

[3] Omar Mossad, Amgad Ahmed, et al., "FAT
ALBERT: Finding Answers in Large Texts using Semantic Similarity Attention Layer based on BERT."

[4] Shervin Malmasi, MarcosZampieri "Detecting Hate Speech in Social- Media"

[5] M. Beskow,Sumeet Kumar, Kathleen M. Carley," The evolution of political memes: Detecting and characterizing internet memes with multi-modal deep learning".

[6] E. S. Smitha , S. Sendhilkumar , and G. S. Mahalaksmi,'Meme Classi- fication Using Textual and Visual Features".

[7] Gargi Ghosh Reshef Shilon, Fan Yang, Xiaochang Peng,Hao Ma ,Eider Moore, Goran Predovic,"Exploring Deep Multimodal Fusion of Text and Photo for Hate Speech Classification".

[8] Alberti, C., Ling, J., Collins, M., Reitter, D.: Fusion of detected objects in text for visual question answering. arXiv preprint arXiv:1908.05054 (2019).

[9] Goswami, V.,Kiela, D., Firooz, H., Mohan, A., Singh, A., Ringshia, P., Testuggine, D.: The hateful memes challenge: Detecting hate speech in multimodal memes. arXiv preprint arXiv:2005.04790 (2020).

[10] Kai Shu, Amy Sliva, Suhang Wang, Jiliang Tang, and Huan Liu. Fake news detection on social media: A data mining perspective. arxiv:1708.01967, 2017.

[11] Anna Schmidt and Michael Wiegand. A survey on hate speech detection using natural language processing. 2017.

[12] Sergey Smetanin. , EmoSense at SemEval-2019, Task 3: Bidirectional LSTM Network for Contextual Emotion Detection in Textual Con- versations. (International Workshop on Semantic Evaluation held in conjunction with NAACL-2019 in New Orleans, LA, USA. )

[13] C. C. Park and G. Kim. Expressing an image stream with a sequence of natural sentences. In Advances in neural information processing systems, pages 73-81, 2015.Paula Fortuna and Sergio Nunes. A survey on automatic detection of hate speech in text. 51(4), 2018.

[14] Agrawal, A., Batra, D., Parikh, D.: Analyzing the behavior of visual question answering models. arXiv preprint arXiv:1606.07356 (2016 